Basic Health Sciences

Poster

Abstract ID: 82

\title{
Clinical and cytogenetic profiles of children with autism in Kuantan Pahang
}

\author{
Nora Mat Zin ${ }^{\mathrm{a}}$ | Naznin Muhammad \\ ${ }^{a}$ Department of Psychiatry, Kulliyyah of Medicine, International Islamic University Malaysia \\ ${ }^{b}$ Department of Pathology and Laboratory Medicine, Kulliyyah of Medicine, International Islamic \\ University Malaysia
}

Introduction: Autism spectrum disorder (ASD) is a group of neurodevelopmental disorder. The aetiology is not known in 75 to 80 percent of patients while in 20 to 25 percent a genetic cause is identified. There are very few studies on cytogenetic profile of ASD being carried out locally. This cross-sectional study was conducted to examine the clinical and cytogenetic profiles of paediatric ASD patients attending the Kuantan branch, National Autistic Society of Malaysia School. Methods: All paediatric patients (up to 18 years of age) attending the school in the year 2009/2010 were recruited. Clinical data was obtained by interviewing the guardians and assessment of the patients. Peripheral blood samples were collected for cytogenetic analysis. Results: Guardians of 14 out of 24 patients (58.3\%) agreed for their children to be enrolled into the study. The age range was 5 to 12 years of age. The diagnostic subgroups were autistic disorder (13/14 patients) and Asperger syndrome (1/14). Approximately $80 \%$ had learning disability and history of allergy. Five patients had hyperactivity and none had history of seizures. There were family histories of developmental delay, mental retardation, epilepsy, psychiatric disorders and allergy. No structural or numerical chromosomal abnormality was detected. Conclusions: Although no cytogenetic abnormalities were identified, most probably due to the small sample size, it is crucial that genetic studies, including molecular genetic be explored as they would provide useful clinical insights and enhance understanding of the pathogenesis.

KEYWORDS: Cytogenetic, Autisme 